\title{
TOPOLOGIES INDUCED BY METRICS \\ WITH DISCONNECTED RANGE
}

\section{Kevin Broughan}

\begin{abstract}
In a metric space $(X, d)$ a ball $B(x, \varepsilon)$ is separated if $d(B(x, \varepsilon), X \backslash B(x, \varepsilon))>0$. If the separated balls form a subbase for the $d$-topology then Ind $X=0$. The metric is gaplike at $x$ if $d_{x}(X)$ is not dense in any neighbourhood of 0 in $[0, \infty)$. The usual metric on the irrational numbers, $\boldsymbol{P}$, is the uniform limit of compatible metrics $\left(d^{n}\right)$, each $d^{n}$ being gap-like on $\mathbb{P}$. In a completely metrizable space $X$ if each dense $G_{\delta}$ is an $F_{\sigma}$ then Ind $X=0$.
\end{abstract}

\section{Introduction}

Earlier results of the author concerning the relationship between the range of a metric and the induced topological structure $[1,2,3,4]$ are extended in this paper.

Let $(X, d)$ be a metric space. It was shown in [3, Theorem 3.4, page 66] that if $d\left(x^{2}\right)$ is not dense in any neighbourhood of 0 in $[0, \infty)$ then Ind $X=0$. The same is true if we assume that $d_{x}(X)$ is not dense in any neighbourhood of 0 for each $x \in X$ where $a_{x}(X)=\{a(x, y): y \in X\}[4$, Theorem 1, page 77]. The results are improved here by reducing the size of the set of points at which the metric range has gaps.

Received 20 August 1981. 
Let $\alpha, \beta$ be in $\mathbb{R}$ with $0<\alpha<\beta$. We say $d$ has a gap $[\alpha, \beta]$ at $x$ in $X$ if $d_{x}(X) \cap[\alpha, \beta]=\varnothing$. We say $d$ has a gap in $[\alpha, \beta]$ at $x$ if there exist $r, s$ with $\alpha \leq r<s \leq \beta$ such that $d$ has a gap $[r, s]$ at $x$. We say the ball $B(x, \varepsilon)$ in $X$ is separated if $d(B(x, \varepsilon), X \backslash B(x, \varepsilon))>0$. We say that the set $B(x, \varepsilon)$ is a gap ball if there is a $\delta>\varepsilon$ such that $d$ has a gap $[\varepsilon, \delta]$ at $x$. The metric $d$ is gap-like at $x$ if $d_{x}(X)$ is not dense in any neighbourhood of 0 in $[0, \infty)$.

It is shown that Ind $X=0$ if the separated or gap balls form a subbase for the $d$-topology (Theorem 1 or Corollary 2 respectively), or if there is a countable family of dense subsets of points at which the metric range has a restricted gap nature (Theorem 3). An example is given to show that this same conclusion is about as false as it can be when the metric is gap like on a single dense set of points. It is then shown how gaps may be introducted into a metric range in the case where $d_{x}(X)$ is not a neighbourhood of 0 in $[0, \infty)$ for all $x$ in $X$. The metric $d$ is the uniform limit of a sequence of compatible metrics for which the set of all points at which at least one is gap-like is dense (Theorem 4). A better result is obtained for $\mathbb{P}$, the irrational numbers, by making an explicit construction for which each metric in the convergent sequence is gap-like on all of $\mathbf{P}$ (Theorem 6). Finally an application of the metric value idea is given to prove that in a completely metrizable space $X$, Ind $X=0$ if each dense $G_{\delta}$ is an $F_{\sigma}$ (Theorem 9).

\section{Gaps in metric values}

THEOREM 1. Let $(X, d)$ be such that the separated balls form a subbase for the topology induced by $d$. Then Ind $X=0$.

Proof. Let $\varepsilon>0$ be given. Let $x \sim y$ if there exists $x_{0}, \ldots, x_{n}$ in $X$ with $x_{0}=x, x_{n}=y$ and $d\left(x_{i-1}, x_{i}\right)<\varepsilon$ for $1 \leq i \leq n$. Let the equivalence classes induced by this relation be denoted by $E(\varepsilon)=(E(x, \varepsilon): x \in X)$ where $x \in E(x, \varepsilon)$. Then each such class is a clopen subset of $X$. If $0<\delta<\varepsilon$ then $E(\delta)$ refines $E(\varepsilon)$.

The covers $(E(I / n): n \in \mathbb{N})$ are clopen and discrete. Let $x \in X$ 
and let $\varepsilon>0$ be given. By hypothesis there are separated balls $B\left(x_{i}, \delta_{i}\right), 1 \leq i \leq n$, satisfying

$$
x \in \prod_{i=1}^{n} B\left(x_{i}, \delta_{i}\right) \subset B(x, \varepsilon) .
$$

Let

$$
B=\min \left\{d\left(B\left(x_{i}, \delta_{i}\right), X \backslash B\left(x_{i}, \delta_{i}\right)\right): 1 \leq i \leq n\right\} .
$$

Then

$$
E(x, \beta) \subset \bigcap_{i=1}^{n} B\left(x_{i}, \delta_{i}\right) .
$$

Therefore the sets $(E(x, \beta): x \in X, \beta>0)$ form a base for the topology generated by $d$ and so by [6, Theorem 5, page 291], Ind $X=0$. $/ /$

COROLLARY 2. If the set of gap balls forms a sub-base for the topology then Ind $X=0$.

Proof. Each gap ball is separate. //

THEOREM 3. Let $(X, d)$ be a metric space. Suppose there exist two sequences $\left(\alpha_{n}\right),\left(B_{n}\right)$ of strictly positive monotonically decreasing real numbers with $\alpha_{n}<\beta_{n}$ for all $n \in N$ and limit $\beta_{n}=0$ such that for each $n$ the set

$$
D_{n}=\left\{x \in X: d \text { has a gap in }\left[\alpha_{n}, B_{n}\right]\right\}
$$

is dense in $X$. Then Ind $X=0$.

Proof. Let $x \in X$ and $\varepsilon>0$ be given. Let $n \in \mathbb{N}$ such that $B_{n}<\varepsilon / 2$. Then there is a $y \in D_{n}$ in $B\left(x, \alpha_{n}\right)$. Since $d$ has a gap in $\left[\alpha_{n}, \beta_{n}\right]$ at $y$ there are real numbers $r$, s with $\alpha_{n} \leq r<s \leq \beta_{n}$ such that $d$ has a gap $[r, s]$ at $y$. Then $B(y, r)$ is a gap ball and $x \in B(y, r) \subset B(x, \varepsilon)$. This shows that the gap balls from a base for the topology generated by $d$ and so, by Corollary 2 , Ind $X=0$. //

One might hope that the conclusion of Theorem 3 would follow if the metric space had a dense subset $D$ with $d_{x}(X)$ not dense in any neighbourhood of 0 in $[0, \infty)$ for each $x$ in $D$. The following example 
shows that given any dimension (finite or transfinite) a metric space with this property can be constructed containing a closed subset having the given dimension. That is to say the hoped for result is very far from being true.

EXAMPLE 4. Let $(X, \tau)$ be any metrizable space with no isolated points. Then there is a metric space $(y, d)$ with $X$ homeomorphic to a closed subspace of $Y$ and an open dense subset $D \subset Y$ with $d_{y}(Y)$ not dense in any neighbourhood of 0 for each $y \in D$.

Let $Z=X \times X$ with the product topology and let $d$ be any compatible metric on $Z$. Let $\Delta=\{(x, x): x \in X\}$ be the diagonal and let $P=Z-\Delta$. Let $D \subset P$ be a maximal subset satisfying

$$
d(x, y) \geq \frac{1}{2} d(x, \Delta)+\frac{1}{2} d(y, \Delta)
$$

for all $x$ and $y$ in $D$ with $x \neq y$. Then by [9, page 111], $\partial P=\Delta$. If $Y=\Delta \cup D$, and we represent the restriction of $d$ to $Y$ by $d$, then $(y, d)$ has the desired properties since, for $x$ in $D$,

$$
d_{x}(Y) \cap[0, d(x, \Delta) / 4]=\{0\}
$$

If $(X, d)$ is such that $d_{x}(X)$ is not a neighbourhood of 0 for each $x$ in some dense set $D$ it is possible to increase the size of the metric gaps at the cost of introducing a denumerable family of compatible metrics.

THEOREM 5. Let $(X, d)$ be a metric space. Let $D \quad X$ be a dense and such that for each $x$ in $D, d_{x}(X)$ is not a neighbourhood of 0 in $[0, \infty)$. Then there exists a family $\left(d^{n}: n \in N\right)$ of compatible metrics on $X$ with the property that for each non-empty open set $P$ there is an $n$ in $\mathrm{N}$ such that $d^{n}$ is gap-like at $x$ for some $x$ in $P$.

Proof. Let $B=\left(B_{n}\right)=\left(B_{\lambda n}: \lambda \in I_{n}, n \in N\right)$ be a o-discrete base for the topology on $X$ generated by $d$. For each $B$ in $B$ choose an $x=x_{B} \in B \cap D$, an $\varepsilon>0$ with $B(x, 2 \varepsilon) \subset B$, and a sequence of distinct points $r_{i} \downarrow 0$ in $\mathrm{R}$ with $r_{1}<\varepsilon, r_{i} \notin d_{x}(X)$, and $2 r_{i+1}<r_{i}$ for all $i$ in $N$. For each $i$ in $X$ let 


$$
f_{B}(y)= \begin{cases}0 & \text { if } y=x \\ r_{i+1} & \text { if } r_{i+1}<d(x, y)<r_{i} \\ 0 & \text { if } r_{1}<d(x, y) .\end{cases}
$$

Then $f_{B}$ is continuous on $X$ and vanishes outside of $B$. Let $n \in \mathbb{N}$. Let

$$
d^{n}(x, y)=d(x, y)+\sum_{B \in B_{n}}\left|f_{B}(x)-f_{B}(y)\right| .
$$

Then each $d^{n}$ is a compatible metric on $X$. For all $y$ in $X$,

$$
d^{n}\left(x_{B}, y\right) \notin\left[r_{i}+r_{i+1}, 2 r_{i}\right] \text { for } i \geq 2 \text {. }
$$

Therefore the family $\left(d^{n}\right)$ has the desired properties.

It is easy to see, by taking completions, that no metric on any dense subset of $R$ can have any gaps in its metric values if it is uniformly equivalent to the usual metric. The following theorem indicates that when the subset is contained in the irrational numbers, the usual metric can be expressed as the uniform limit of a sequence of compatible metrics having gaps at each point.

LEMMA 6. Let $\mathbf{P}$ be the irrational numbers. Then there exists a compatible metric $\rho$ on $\mathbf{P}$ such that $\rho$ is gap-like on $\mathbf{P}$ and such that

$$
|\rho(x, y)-| x-y|| \leq 1
$$

for each $x$ and $y$ in $\mathbf{P}$.

Proof. For $k \in \mathbb{Z}$ and $n \in \mathbb{N} \cup\{0\}$ let $\xi(k, n)=k .2^{-n}$. Let $D(k, n)=[\xi(k, n), \xi(k+1, n)] \cap \mathbf{P}$. Then $(D(k, n): k \in \mathbb{Z}, n \in \mathbb{N} \cup\{0\})$ is a sieve for $P([2])$ and generates the usual topology.

$$
\begin{aligned}
& \text { If } x \text { and } y \text { are in } P \text { let } \\
& B(x, y)=\left\{\begin{array}{l}
0 \text { if } x=y, \\
\min \left\{2^{-n}: x \text { and } y \text { are both in } D(k, n) \text { for some } k\right\}, \\
1 \text { if } x \text { and } y \text { are in no common } D(k, n) .
\end{array}\right.
\end{aligned}
$$

Then $\beta$ is a compatible metric on $\mathbf{P}$. Define another compatible metric by 


$$
\rho(x, y)=|x-y|+\beta(x, y) \text {. }
$$

Then $|\rho(x, y)-| x-y|| \leq 1$ for all $x$ and $y$ in $\mathbb{P}$.

Let $y \in \mathbb{P}$ be fixed. Then $y=n+\sum_{i=1}^{\infty} \varepsilon(i) 2^{-i}$ where $\varepsilon(i) \in\{0,1\}$ for each $i$ and $n \in \mathbb{Z}$. We will prove the following claim: there exists an infinite subsequence $(\varepsilon(f(i)))$ such that for all $i$ in $N$, $\varepsilon(f(i))=0$ and $\varepsilon(f(i)+1)=0$ or an infinite subsequence $(\varepsilon(g(i)))$ such that $\varepsilon(g(i))=1$ and $\varepsilon(g(i)+1)=1$ for all $i$ in $N$. If not, either $\varepsilon(2 i)=1$ and $\varepsilon(2 i+1)=0$ or $\varepsilon(2 i)=0$ and $\varepsilon(2 i+1)=1$ for all $i$ sufficiently large. In either case $y \in P$ which is impossible. Hence a subsequence of one or other of the different forms must exist.

Let $n>0$ be given. Let $i$ in $N$ be such that $i \geq 3$ and $3.2^{-i}<\eta$. Let $\varepsilon(i)=1$ and $\varepsilon(i+1)=1$. (The proof when $\varepsilon(i)=0$ and $\varepsilon(i+1)=0$ is similar and is omitted.)

There is a $k$ in $\mathbb{Z}$ with $\xi(k, i-2)<y<\xi(k+1, i-2)$. Assume $\varepsilon(i-1)=0$ and $\varepsilon(i+2)=0$. The proof for the three other possibilities is omitted. Then $\xi(8 k+3, i+1)<y<\xi(2 k+1, i-1)$. Let $E=\mathbf{P} \cap[\xi(k, i-2), \xi(k+1, i-2)]$. Divide $E$ into four disjoint intervals $A, B, C$ and $D$ defined below:

$$
\begin{aligned}
A & =\mathbf{P} \cap[\xi(2 k+1, i-1), \xi(k+1, i-2)], \\
B & =\mathbf{P} \cap[\xi(k, i-2), \xi(4 k+1, i)], \\
C & =\operatorname{P} \cap[\xi(4 k+1, i), \xi(8 k+3, i+1),
\end{aligned}
$$

and

$$
D=\mathbb{P} \cap[\xi(8 k+3, i+1), \xi(2 k+1, i-1)] .
$$

If $x \in A$ then $\beta(x, y)=4.2^{-i} ;$ if $x \in B$ then $\beta(x, y)=2.2^{-i}$, if $x \in C$ then $\beta(x, y)=2^{-i}$; and if $x \in D$ then $\beta(x, y) \leq 2^{-i+1}$.

The following inequalities are true in the given subsets:

$$
\begin{aligned}
& \text { if } x \in A, \rho(x, y)>y-(4 k+1) 2^{-i}+2.2^{-i} ; \\
& \text { if } x \in B, \rho(x, y) \geq y-(4 k+1) 2^{-i}+2.2^{-i} ; \\
& \text { if } x \in A, \rho(x, y) \leq y-(4 k+1) 2^{-i}+2^{-i} ;
\end{aligned}
$$


if $x \in D, \rho(x, y)<y-(4 k+1) 2^{-i}+2^{-i}$.

If $x \notin E$ then $B(x, y) \geq 2^{-i+2}$ and therefore

$$
\rho(x, y)>2^{-i+2}>3.2^{-i} \text {. }
$$

From these inequalities it follows that

$$
\rho_{y}(\mathbf{P}) \cap\left[y-(4 k+1) 2^{-i}+2^{-i}, y-(4 k+1) 2^{-i}+2 \cdot 2^{-i}\right]=\emptyset \text {. }
$$

Because $y-(4 k+1) 2^{-i}<2^{-i}$ the right hand end point of the above missing interval is less than $3.2^{-i}$ and $3.2^{-i}<n$ be choice of $i$. Hence $\rho_{y}(\mathbf{P})$ is not dense in any neighbourhood of $0 . / /$

THEOREM 7. There exists a sequence $\left(d^{n}\right)$ of compatible metrics on $\mathbb{P}$ such that, for each $x$ in $\mathbb{P}$ and $n$ in $N, d_{x}^{n}(\mathbb{P})$ is not dense in any neighbourhood of 0 in $[0, \infty)$, and such that $d^{n}(x, y) \rightarrow|x-y|$ uniformly on $\mathbf{P}$.

Proof. By Theorem 4 there is a compatible metric $\rho$ on $\mathbb{P}$ with $|\rho(x, y)-| x-y|| \leq 1$ for $x, y$ in $\mathbb{P}$. Then, if $n \in \mathbb{N}$, $|\rho(n x, n y)-| n x-n y|| \leq 1$, and therefore $|(1 / n) \rho(n x, n y)-| x-y|| \leq 1 / n$.

Let $d^{n}(x, y)=(1 / n) \rho(n x, n y)$. Then the sequence $\left(d^{n}\right)$ has the desired properties. $\quad / /$

\section{$G_{\delta}$ supersets of zero dimensional subsets}

In the following lemma a new proof is presented of the result that every subset having large inductive dimension $n$ of less of a metrizable space has a $G_{\delta}$ superset also having dimension $n$ or less $[7$, Theorem 1.4, page 241].

LEMMA 8. Let $(X, \tau)$ be metrizable and let $A \subset X$ have Ind $A \leq n$. Then there is a $G_{\delta}$ subset $B$ of $X$ with $A \subset B$ and Ind $B \leq n$.

Proof. If $A \neq \emptyset$ and Ind $A=0$ there is a compatible metric $d$ on $A$ with values in $H=\{1 / n: n \in \mathbb{N}\} \cup\{0\},[1]$. The metric $d$ may be 
extended to a compatible metric $\hat{d}$ on a $G_{\delta}$ superset of $A$ with $A \subset B \subset \operatorname{cl}(A)$ [10, Problem 24M, page 185]. Necessarily $\hat{d}(B \times B) \subset H$. Therefore Ind $B=0$. If Ind $A \leq n$ there exist $A_{0}, \ldots, A_{n}$ in $X$ with $A=A_{0} \cup \ldots \cup A_{n}$ and Ind $A_{i} \leq 0$ for all $i$. For each $i$ let $B_{i}$ be a $G_{\delta}$ superset of $A_{i}$ with Ind $B_{i}=0$. Then $B=B_{0} \cup \ldots \cup B_{n}$ is a $G_{\delta}$ superset of $A$ and Ind $B \leq n$. //

The following result guarantees the existence of a reasonably large zero dimensional subset in any metrizable space.

LEMMA 9. Let $(X, \tau)$ be metrizable. Then there is a dense $G_{\delta}$ subset $B$ of $X$ with Ind $B=0$.

Proof. Let $d$ generate $\tau$. For each $n$ in $N$ let $D_{n}$ be a maximal subset of $X$ with $D_{n+1} \supset D_{n}$ and $d(x, y) \geq 1 / n$ for each $x$ and $y$ in $D_{n}$ with $x \neq y$. Then $A=\bigcup_{n=1}^{\infty} D_{n}$ is $\sigma$-discrete and hence Ind $A=0$. Let $B$ be a $G_{\delta}$ superset of $A$ with Ind $B=0$. Since $A$ is dense [5, page 168], so also is $B$. //

Using Lemma 9 we can prove a theorem similar in spirit to the following result of Reed: if we assume Goedel's axiom of constructibility (often abbreviated to $V=L$ ) then any normal and first countable space in which every subset is an $F_{\sigma}$ is necessarily $\sigma$-discrete [8, page 46]. We obtain the following result without making any special set theoretical assumptions.

THEOREM 10. Let $(X, T)$ be completely metrizable and such that each dense $G_{\delta}$ subset is an $F_{\sigma}$. Then Ind $X=0$.

Proof. Let $P=U\{Q \in \tau$ : Ind $Q=0\}$. Then by [7, Theorem 4.12, page 173], Ind $P=0$. If $Y=X-P$ is non-empty then $Y$ is completely metrizable and has no non-empty open subsets having large inductive dimension zero. Let $B \subset Y$ be a dense (in $Y$ ) $G$ subset of $Y$ having Ind $B=0$. Then $B$ is $G_{\delta}$ in $X$ and hence, by hypothesis, $B=\bigcup_{i=1}^{\infty} F_{i}$ 
for some family $\left(F_{i}\right)$ of closed subsets of $Y$. Since $B$ is $G_{\delta}$ and dense in $Y, Y-B=\bigcup_{j=1}^{\infty} H_{j}$ for some family $\left(H_{j}\right)$ of closed subset of

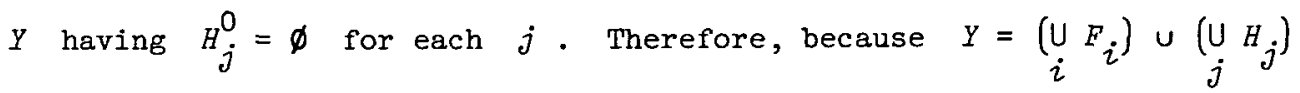
and is Baire there is an $i$ in $\mathrm{N}$ with $F_{i}^{0} \neq \varnothing$. But then, Ind $F_{i}^{0}=0$ since $F_{i} \subset B$. This contradicts the assumption that $Y$ has no non-empty subsets with dimension zero and therefore the assumption that $Y$ is nonempty. Hence Ind $X=0$. //

\section{References}

[1] K.A. Broughan, "A metric characterizing Čech dimension zero", Proc. Amer. Math. Soc. 39 (1973), 437-440.

[2] K.A. Broughan, "Metrization of spaces having Čech dimension zero", BuzZ. Austral. Math. Soc. 9 (1973), 161-168.

[3] Kevin A. Broughan, Invariants for real-generated uniform topological and algebraic categories (Lecture Notes in Mathematics, 491. Springer-Verlag, Berlin, Heidelberg, New York, 1975).

[4] Kevin Broughan, "Four metric conditions characterizing Čech dimension zero", Proc. Amer. Math. Soc. 64 (1977), 176-178.

[5] W.W. Comfort, "A survey of cardinal invariants", General Topology App I. 1 (1971), 163-199.

[6] R. Engelking, Outzine of general topology (translated by $K$.

Sieklucki. North-Holland, Amsterdam; PWN - Polish Scientific

Publishers, Warsaw; 1968).

[1] A.R. Pears, Dimension theory of general spaces (Cambridge University Press, Cambridge, New York, Melbourne, 1975).

[8] Mary Ellen Rudin, Lectures on set theoretic topology (Conference Board of the Mathematical Sciences, Regional Conference Series in Mathematics, 23. American Mathematical Society, Providence, Rhode Island, 1975). 
[9] Russell C. Walker, The Stone-Čech compactification (Ergebnisse der Mathematik und der Grenzgebiete, 83. Springer-Verlag, Berlin, Heidelberg, New York, 1974).

[10] Stephen Willard, General topology (Addison-Wesley, Reading, Massachusetts; London; Don Mills; 1970).

Department of Mathematics,

University of Waikato,

Private Bag,

Hami Iton,

New Zealand. 